\title{
Cartap hydrochloride poisoning: A clinical experience
}

\author{
Hari K. Boorugu, Anugrah Chrispal
}

Cartap hydrochloride, a nereistoxin analog, is a commonly used low toxicity insecticide. We describe a patient who presented to the emergency department with alleged history of ingestion of Cartap hydrochloride as an act of deliberate self-harm. The patient was managed conservatively. To our knowledge this is the first case report of Cartap hydrochloride suicidal poisoning. Cartap toxicity has been considered to be minimal, but a number of animal models have shown significant neuromuscular toxicity resulting in respiratory failure. It is hypothesized that the primary effect of Cartap hydrochloride is through inhibition of the $\left[{ }^{3} \mathrm{H}\right]$-ryanodine binding to the $\mathrm{Ca}^{2+}$ release channel in the sarcoplasmic reticulum in a dose-dependent manner and promotion of extracellular $\mathrm{Ca}^{2+}$ influx and induction of internal $\mathrm{Ca}^{2+}$ release. This results in tonic diaphragmatic contraction rather than paralysis. This is the basis of the clinical presentation of acute Cartap poisoning as well as the treatment with chelators namely British Anti Lewisite and sodium dimercaptopropane sulfonate.

Keywords: Cartap hydrochloride, nereistoxin, respiratory depression

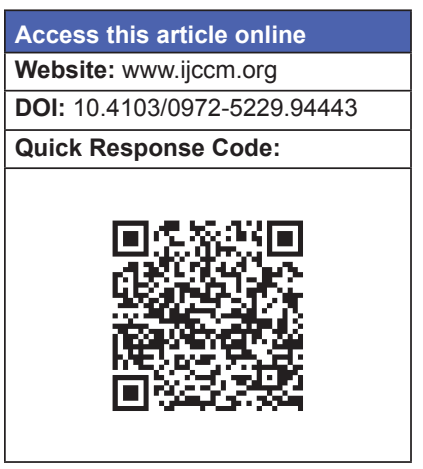

\section{Introduction}

Cartap is a pesticide that was first introduced into the market in Japan in 1967. Its commercial names include Padan, Kritap, AG-Tap, Thiobel, and Vegetox. Its basic chemical structure is S, S-[2-(dimethylamino)-1, 3 -propanediyl] dicarbamothioate [Figure 1]. It is commonly used as a hydrochloride $\left(\mathrm{C}_{7} \mathrm{H}_{15} \mathrm{~N}_{3} \mathrm{O}_{2} \mathrm{~S}_{3} \mathrm{HCl}\right)$. Cartap is essentially a contact and stomach poison. It is used for the control of chewing and sucking pests and results in insect paralysis. It has been categorized as a high-effectiveness, low-toxicity, and low-residue pesticide used in rice and sugarcane fields. It is generally considered to be a safe compound with oral $\mathrm{LD}_{50}$ in the monkey of $100--200 \mathrm{mg} / \mathrm{kg}$ body weight. ${ }^{[1]}$ It was believed that it causes little skin or ocular irritation on exposure. However, subsequent studies have shown

From:

Department of Medicine Unit 2, Christian Medical College, Vellore,

Tamil Nadu, India

\section{Correspondence:}

Dr. Hari Kishan Boorugu, Department of Medicine Unit 2, Christian Medical College and Hospital, Vellore- 632 004, Tamil Nadu, India.

E-mail: drharikishan@gmail.com that ocular instillation can result in diaphragmatic contracture and death in rabbits. ${ }^{[2]}$ We report a case of intentional ingestion of Cartap hydrochloride as a suicidal attempt in a farmer which subsequently resulted in severe respiratory failure.

\section{Case Report}

A 27-year-old male agricultural laborer from Tamil Nadu, India, presented with alleged consumption of $50 \mathrm{ml}$ of an unknown poison as an act of deliberate self-harm following a quarrel with his spouse. Following consumption he had several episodes of vomiting. Subsequently, he developed twitching movements of his limbs, urinary incontinence, and altered sensorium. He was brought to the Emergency Department 4 hours after consumption. On examination he was found to be stuporous (GCS 11/15). He had a systolic blood pressure of $80 \mathrm{~mm}$ of $\mathrm{Hg}$, a heart rate of $120 / \mathrm{min}$, and

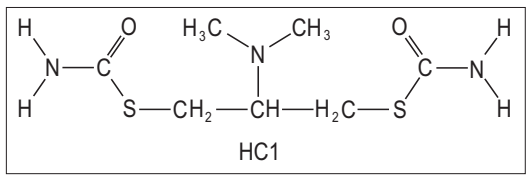

Figure I: Chemical structure of Cartap hydrochloride 
a respiratory rate of $32 / \mathrm{min}$. He was documented to have hypoxia (pulse oximetry saturation of $70 \%$ ). The pupillary size was $3 \mathrm{~mm}$ bilaterally and reacting to light. He was noted to have occasional fasciculations. Systemic examination was otherwise unremarkable. He was intubated and mechanically ventilated in view of altered sensorium and hypoxia. A gastric lavage was administered followed by a single dose of activated charcoal instilled through the nasogastric tube. The provisional diagnosis made in the emergency department was of an organophosphate poisoning with acute cholinergic syndrome. The chest X-ray was normal and ECG revealed sinus tachycardia. Basic blood investigations including serum electrolytes, creatinine, liver function tests, and complete blood counts were normal. The serum pseudo-cholinesterase level was normal (3839 U/1). The patient was admitted into the semiintensive care unit and managed conservatively with fluids and cardiac monitoring. The plan was to use atropine if the patient developed bradycardia or miosis and to repeat serum pseudo-cholinesterase levels after 24 hours.

Twenty-four hours after presentation, the relatives were able to provide the name and container of the poison which was Cartap hydrochloride $4 \mathrm{gm} / 50 \%$ SP. At 24 hours the patient had shown_improvement in sensorium and ventilatory supports were rapidly reduced. He was subsequently extubated. Due to the fact that there is insufficient data regarding effective timing of the antidote, and the significant clinical recovery of our patient, the antidote was not administered. The patient was discharged after counseling 3 days after admission with no complications.

\section{Discussion}

Cartap has been recognized as an analog of nereistoxin which is a neurotoxic substance initially isolated from the annelid Lumbriconereis heteropoda. It is however believed that Cartap is of a lower potency to nereistoxin. The other nereistoxin analogs that are in use are bensultap, thiocyclam, and thiosultap. Its primary action was earlier considered to be by direct action on and inhibition of the postsynaptic nicotinic acetylcholine receptor (AChR) ion channels. Due to this action it was believed that Cartap hydrochloride would act as a neuromuscular blocker and hence result in respiratory paralysis in the setting of acute toxicity. A study published by Liao et al. challenges this belief based on studies of ocular Cartap exposure in mice and its action on the phrenic nerve. The conclusion was that the effect on the diaphragm was not due to neuromuscular paralysis but instead due to persistent diaphragmatic contraction resulting in respiratory failure. ${ }^{[1]}$
Cartap may exert this effect primarily by promoting extracellular $\mathrm{Ca}^{2+}$ influx and induction of internal $\mathrm{Ca}^{2+}$ release. Cartap inhibits the $\left[{ }^{3} \mathrm{H}\right]$-ryanodine binding to the $\mathrm{Ca} 2+$ release channel in the sarcoplasmic reticulum in a dose-dependent manner. It was hypothesized that Cartap-induced contracture was, to a minor extent, a result of inhibition of the sarcoplasmic reticulum $\mathrm{Ca}^{2+}$ pump protein $\mathrm{Ca}^{2+}$ ATPase. Inhibition of the ATPase would result in the unloading of calcium from the sarcoplasmic reticulum. ${ }^{[1]}$

There have been a few reports of human toxicity in Japan and India as part of occupational exposure. Acute toxicity of the compound that has been described is nausea, vomiting tremulousness, salivation, spasms, dyspnea, and mydriasis. Sodium dimercaptopropane sulfonate (DMPS) and sodium dimercaptosuccinate (DMS) were discovered to be effective antidotes for acute poisoning of insecticides like SCD [sodium ammonium dimethyl-2-(propane-1, 3-dithiosulfate) monohydrate], nereistoxin, and Cartap in mouse models. ${ }^{[3]}$ These antidotes completely antagonized the respiratory depression caused by these compounds. Cysteine was found to be of lower effectiveness and shorter duration of action than these compounds. At present the recommended antidotes for Cartap poisoning are an intravenous injection of $100-200 \mathrm{mg}$ of L-cysteine or an intramuscular injection of $20-60 \mathrm{mg}$ of British Anti Lewisite (Dimercaprol; 2, 3-dimercapto propanol). It is possible that the effect of calcium binding of these antidotes is central to the antagonistic effects on respiratory depression in Cartap toxicity.

To the best of our knowledge this is the first reported case of Cartap hydrochloride poisoning as a result of deliberate self-harm that manifested with acute respiratory distress and altered sensorium. The patient was managed conservatively without the antidote and recovered with no complications.

\section{References}

1. Liao JW, Kang JJ, Liu SH, Jeng CR, Cheng YW, Hu CM, et al. Effects of cartap on isolated mouse phrenic nerve diaphragm and its related mechanism. Toxicol Sci 2000;55:453-9.

2. Liao JW, Pang VF, Jeng CR, Chang SK, Hwang JS, Wang SC. Susceptibility to cartap-induced lethal effect and diaphragmatic injury via ocular exposure in rabbits. Toxicology 2003;5;192:139-48.

3. Cao BJ, Chen ZK, Chi ZQ. [Antidotal effects of sulfhydryl compounds on acute poisonings by sodium ammonium dimethyl-2-(propane-1,3dithiosulfate) monohydrate, nereistoxin and cartap]. Zhongguo Yao Li Хue Вao 1990;11:180-4.

How to cite this article: Boorugu HK, Chrispal A. Cartap hydrochloride poisoning: A clinical experience. Indian J Crit Care Med 2012;16:58-9.

Source of Support: Nil, Conflict of Interest: None declared. 